\title{
Diferente Asimetría Craneal Según Sexo en el Conejo Toy
}

\author{
Different Skull Asymmetry According to Sex in Toy Rabbits
}

Parés-Casanova, P. M.

\begin{abstract}
PARÉS-CASANOVA, P. M. Diferente asimetría craneal según sexo en el conejo toy. Int. J. Morphol., 39(3):864-868, 2021.
RESUMEN: Los conejos denominados toy son seleccionados por su apariencia pedomórfica ('infantilizada'). El objetivo principal de este estudio fue analizar las asimetrías craneales en este tipo de conejo. Para ello, se estudiaron un total de 46 cráneos adultos (9 machos y 37 hembras), recurriéndose a técnicas de morfométrica geométrica a partir 10 hitos anatómicos pareados y 3 en el plano sagital, en la cara dorsal del cráneo. Nuestra hipótesis es que a través de la selección artificial en los machos (objetivo principal para la obtención de nuevos fenotipos) las asimetrías se expresarán como valores de asimetría más altos. El tamaño de los cráneos resultó similar entre ambos sexos, pero presentaban la asimetría fluctuante fue mayor en machos. La asimetría fluctuante se considera un indicador negativo de la capacidad de resistir pequeñas anomalías en su desarrollo, anomalías que son generalmente el resultado de estrés genético o ambiental. En otras palabras, los machos estarían sujetos a una selección más fuerte y, en consecuencia, se verían más afectados por el manejo, en comparación con las hembras. Estos resultados pueden facilitar la comprensión de los patrones y procesos subyacentes al dimorfismo sexual en fenotipos extremos, como lo son los de los conejos toy.
\end{abstract}

PALABRAS CLAVE: Asimetría bilateral; Oryctolaguscuniculus; Osteología; Pedomorfia; Dimorfismo sexual.

\section{INTRODUCCIÓN}

Una de las utilidades del análisis con métodos de morfometría geométrica es su capacidad de detectar alteraciones de la simetría (Mardia et al., 2000; Briones \& Guiñez, 2008). Entre los diversos tipos de asimetría, hay la asimetría fluctuante (AF), caracterizada por desviaciones sutiles y aleatorias de la simetría en rasgos bilateralmente simétricos (Briones \& Guiñez), y la asimetría direccional (AD), que se caracteriza por una distribución sesgada hacia uno de los lados (derecho o izquierdo) a nivel poblacional y que se origina como respuesta a estímulos externos que afectan diferencialmente a ambos lados del organismo (Lotto \& Beguelin, 2014). La AF se considera un indicador negativo de la capacidad de resistir pequeñas anomalías en su desarrollo, anomalías que son generalmente el resultado de estrés genético o ambiental (Zachos et al., 2007; Graham et al., 2010). La AD se considera con una base genética significativa (Palmer, 1994) y, por lo tanto, sería apropiada para la estimación de procesos adaptativos.

Los animales domésticos han sido seleccionados en favor de un perfil juvenil de la cabeza, hocico corto y otros rasgos. Además, la retención de rasgos juveniles hace que los animales sean más tratables y fáciles de manejar. Los cambios físicos también se relacionan con cambios en el comportamiento. La retención de rasgos juveniles en la adultez se conoce como pedomorfia, y se debe sobre todo a una cara proporcionalmente corta en relación al cráneo, que confiere una apariencia más atractiva como animal de compañía (Goodwin et al., 1997). Los conejos toy presentan un aspecto pedomórfico, infantilizado, resultado de una selección artificial encaminada a buscar fenotipos de aspecto infantil, conejos que como mascota sean más preferidos en el mercado.

En la investigación actual, nuestro objetivo era detectar posibles diferencias en la asimetría craneal en conejos toy. Hasta hoy, y por lo menos hasta donde sabemos, no ha habido ninguna investigación para estudiar las diferencias sexuales en las asimetrías craneales del conejo toy, aunque sí para los humanos (Graham et al.). Este estudio permitirá una mayor comprensión de los fenómenos de estrés bajo el proceso de formación de razas o variedades animales domésticas.

\section{MATERIAL Y MÉTODO}

Muestra. El material de este estudio proviene de la mo- 
derna colección esquelética documentada originaria del Laboratorid'Anatomia Animal del Departament de Ciència Animal de la Universitat de Lleida, que incluía cráneos intactos de conejo toy de 46 individuos adultos ( 9 machos y 37 hembras).

Fotografías y obtención de los datos. Se obtuvieron fotografías digitales en la cara dorsal de cada cráneo, situando el eje focal de la cámara, paralelo a esta cara. La cámara estaba conectada a una columna con un brazo ajustable, en un zócalo de rejilla para referencia de medida. La captura de imágenes se realizó con una cámara digital Nikon® D70 (Nikon Inc., Tokio, Japón) (resolución de imagen de 2.240 x 1.488 píxeles) equipada con un teleobjetivo Nikon AF Nikkor® de 28 a 200 mm (Nikon Inc., Tokio, Japón). El estudio morfométrico se basó en un conjunto de 10 hitos laterales (en 5 pares por lado) y 3 en el plano sagital (Fig. 1). Estos puntos consideramos que dan una buena representación de la forma general del cráneo y de una
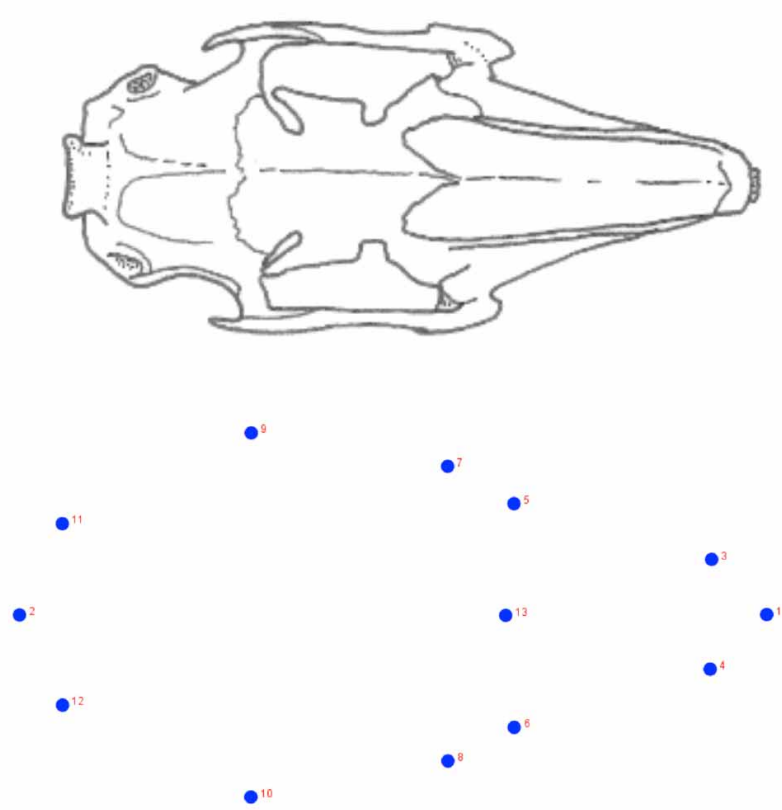

Fig. 1. Conjunto de los hitos anatómicos (5 pares por lado y 3 en el plano sagital) utilizados para este estudio. Cubrieron tanto el neurocráneo (hitos 7 a 12) como el viscerocráneo (hitos 1 a 6, y 13).

manera que nos permite ver características importantes de la asimetría.

Cada punto de referencia se digitalizó dos veces de forma independiente para permitir la estimación de la replicabilidad. Las imágenes capturadas se transformaron en el software TpsUtil v. 1.40 (Rohlf, 2015). Las coordenadas Cartesianas $\mathrm{X}$ e $\mathrm{Y}$ de todos los puntos de referencia se digitalizaron utilizando el software TpsDig v. 2.26
(Rohlf). Este conjunto fue estandarizado aún más por el Análisis Generalizado de Procrustes (AGP). El AGP superpone cada figura al centroide (punto medio de una configuración de los hitos), desplazando posteriormente las figuras a fin de minimizar las distancias cuadradas entre los mismos hitos, obtuviéndose finalmente las coordenadas de Procrustes.

Análisis estadístico. La replicabilidad de las coordenadas Procrustes fue analizada por un NPMANOVA de dos vías con individuos y réplicas como factores. Para detectar asimetrías, la interacción entre el lado e individuo correspondía a la AF y el efecto del lado correspondía a la AD. El error de medición, correspondiente al factor residual, representa el componente de la varianza global debido a la imprecisión de las mediciones. Se hizo un segundo NPMANOVA para cada sexo por separado para detectar las diferencias sexuales de la AF.El tamaño se consideró a partir del "tamaño de centroide", calculado como la raíz cuadrada de la suma de las distancias euclidianas cuadradas desde cada punto de referencia hasta el centroide del espécimen, y proporcionando la estimación del tamaño (Bookstein, 1991). También se hizo una correlación lineal (con el tamaño de centroide log-transformado como variable independiente) del tamaño centroide del cráneo con el componente asimétrico. La comparación de los tamaños centroide entre sexosse realizó con una prueba de Mann-Whitney U. Luego se aplicó un Análisis Canónico Variante (ACV) a partir de pruebas de permutación (10000 rondas de permutación) para las distancias Mahalanobis entre ambos sexos para detectar diferencias estadísticamente significativas para el componente asimétrico. Finalmente se realizó un Análisis de Componentes Principales (ACP) para evaluar la contribución de cada uno de los hitos ala asimetríaglobal del cráneo.Se compararon los patrones de covariación para el módulo neurocraneal (hitos 7 a 12) y para el módulo esplacnocraneal (hitos 1 a 6 , y 13), los cuales fueron cuantificados usando el coeficiente de RV (Klingenberg, 2014). Los análisis estadísticos se realizaron con los programas MorphoJ v. $1.06 \mathrm{c}$ (Klingenberg, 2011) y PAST v. $2.17 \mathrm{c}$ (Hammer et al., 2001).

Declaración de ética. Este estudio se llevó a cabo en piezas óseas de una colección existente, por lo que ningún acuerdo del Comité de ética se consideró innecesario.

\section{RESULTADOS}

La cantidad individual de variación excedió la variación de las réplicas, que presentaron únicamente un 3,3\%, 
inferior además a la variación de las otras fuentes (Tabla I), lo que sugiere que el error de medición, y por ende la replicabilidad, no afectó al resultado de los análisis de asimetría. La AF apareció más elevada para los machos que para las hembras, un $7,72 \%$ frente a un $4,18 \%$ respectivamente (Tabla II). La correlación con el componente asimétrico fue significativa ( $\mathrm{p}=0,0082$ ), explicando el cambio del tamaño un $1,80 \%$ del cambio en la asimetría craneal (Fig. 2), aunque los tamaños centroide del cráneo no estadísticamente diferentes ( $U=572, p=0,357)$ (Fig. 3). El ACV reflejó las diferencias estadísticas entre los sexos ( $\mathrm{p}=0,0127)$. En relación a los resultados del ACP (Tabla III), los tres primeros componentes principales representaron un total del $74,4 \%$ de la varianza observada. El primer componente aportó el 47,7 \%, el segundo, el 16,8 \% y el tercero, el 9,7 \%, de la variación total observada. La mayoría de los hitos más informativos fueron los asociados a la longitud del viscerocráneo (X1, X3, X4 y X13). La cuadrícula de deformación indica que la asimetría se manifiesta sobretodo en la amplitud de la base facial y en el alargamiento nasal (Fig. 4). El RV fue de 0,15, de lo que se deduce un crecimiento modular no isométrico.

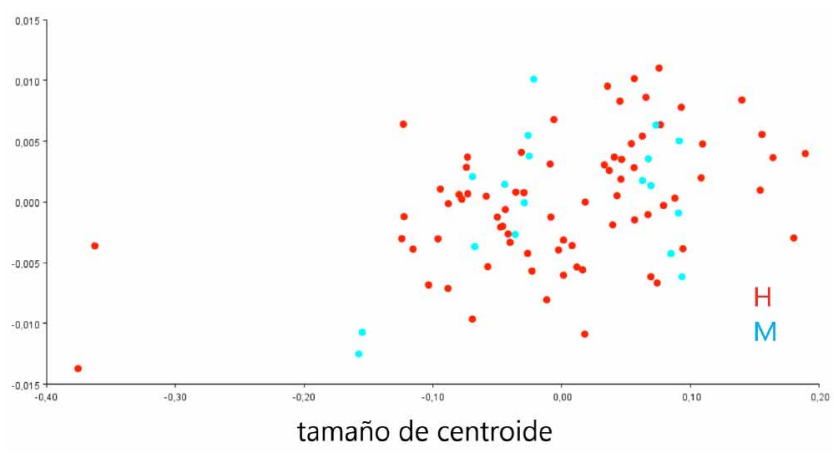

Fig. 2. Regresión entre el componente asimétrico y el tamaño de centroide (log-transformado) para machos $(\mathrm{M}, \mathrm{n}=9)$ y hembras $(\mathrm{H}$, $\mathrm{n}=37$ ) de conejos toy. La correlación con el componente asimétrico fue significativa, explicando pues el tamaño un 1,80\% del cambio en asimetría.

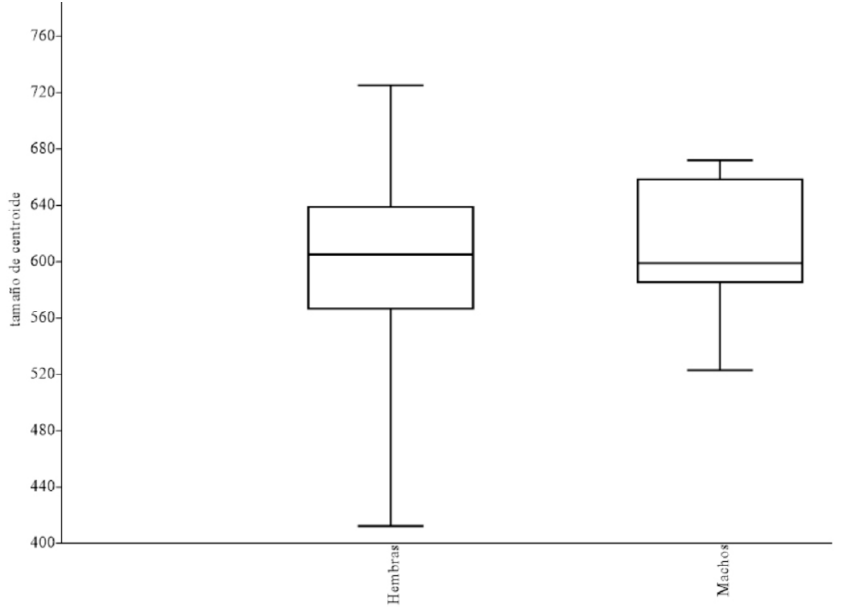

Fig. 3. Diagrama de cajón para los tamaños de centroide del cráneo para machos $(n=9)$ y hembras $(n=37)$ de conejos toy. La mediana de viene representada por el valor central dentro de cada caja, que representa el $50 \%$ central de las puntuaciones de una variable, sin el $25 \%$ más pequeño y el $25 \%$ más alto representados por los "bigotes" superior e inferior. Los tamaños centroide no presentaron diferencias estadísticamente significativas.

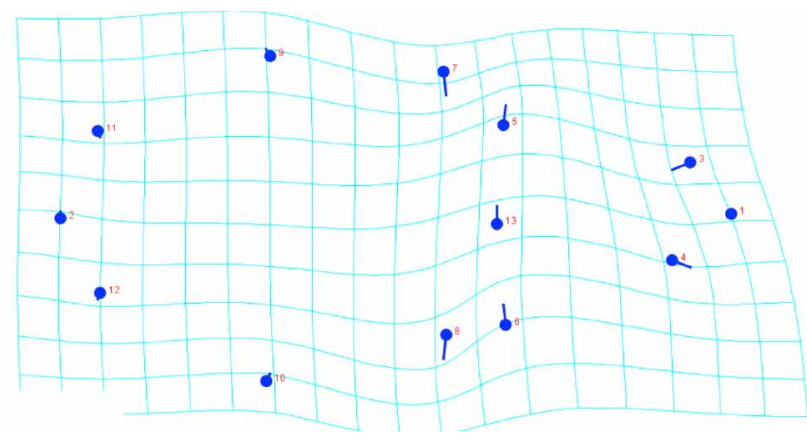

Fig. 4. La cuadrícula de deformación. La asimetría se manifestó básicamente en la amplitud de la base facial y en el alargamiento nasal.

Tabla I. NPM ANOVA de dos vías utilizando 9999 permutaciones y distancias euclidianas para las 10 coordenadas Procrustes de cráneo (cara dorsal) para 46 conejos toy. La cantidad individual de variación individual excedió claramente la variación de las otras fuentes, lo que sugiere que el error de medición fue aleatorio y no afectó los resultados de asimetría.

\begin{tabular}{|c|c|c|c|c|c|}
\hline Fuente & Sumatorio de cuadrados & Cuadrado medio & Grados de libertad & $F$ & $p$ \\
\hline Individuo & 0,11156625 & 0,0002253864 & 495 & 11,0 & $<, 0001$ \\
\hline Lado & 0,00236757 & 0,0002152340 & 11 & 10,5 & $<, 0001$ \\
\hline Interacción lado*individuo & 0,01010675 & 0,0000204177 & 495 & 1,2 & 0,0004 \\
\hline Residual (réplicas) & 0,01597907 & 0,0000157896 & 1012 & & \\
\hline
\end{tabular}


Tabla II. NPM ANOVA de dos vías utilizando 9999 permutaciones y distancias euclidianas para las coordenadas Procrustes del cráneo (aspecto dorsal), con individuos y réplicas como factores para conejos machos $(\mathrm{n}=9)$ y hembras $(\mathrm{n}=37)$. Las asimetrías fluctuantes ("Interacción lado*individuo") (en negrita) fueron significativas para ambos sexos, apareciendo más altas en los machos (7,7 \%) que en las hembras $(4,2 \%)$.

\begin{tabular}{lccccc}
\hline Fuente & Sumatorio de cuadrados & Cuadrado medio & Grados de libertad & $F$ & $p$ \\
\hline Individuo & 0,018910 & 0,0002148857 & 88 & 9,4 & $<, 0001$ \\
Lado & 0,000467 & 0,0000424101 & 11 & 1,6 & 0,0559 \\
Interacción lado*individuo & 0,002007 & $\mathbf{0 , 0 0 0 0 2 2 8 1 1 0}$ & 88 & 1,4 & 0,0116 \\
Residual (réplicas) & 1 & 0,0000153070 & $1,53 \mathrm{E}-05$ & 198 & \\
\hline Fuente & Sumatorio de cuadrados & Cuadrado medio & Grados de libertad & $F$ \\
\hline Individuo & 0,088957 & 0,0002246401 & 396 & 11,5 & $<, 0001$ \\
Lado & 0,002273 & 0,0002066234 & 11 & 10,5 & $<, 0001$ \\
Interacción lado*individuo & 0,00773 & $\mathbf{0 , 0 0 0 0 1 9 5 2 0 2}$ & 396 & 1,2 & 0,0083 \\
Residual (réplicas) & 1 & 0,0000159100 & $1,59 \mathrm{E}-05$ & 814 \\
\hline
\end{tabular}

Tabla III. Valores de descarga para los componentes principales (CP) 1, 2 y 3, para el componente asimétrico.El CP1 explicó un $47,7 \%$, el CP2 un 16,8\% y el CP3 un $9.7 \%$, de la variación total observada.Los valores $<[0.3]$ aparecen en negrita.

\begin{tabular}{lrrr}
\hline & PC1 & PC2 & PC3 \\
\hline $\mathbf{x 1}$ & $\mathbf{- 0 . 3 4 5 6 5 5}$ & 0.026648 & 0.164965 \\
y1 & 0.000000 & 0.000000 & 0.000000 \\
x2 & -0.063044 & -0.005014 & 0.241587 \\
y2 & 0.000000 & 0.000000 & 0.000000 \\
x3 & $\mathbf{- 0 . 3 6 0 5 6 0}$ & -0.094028 & -0.007491 \\
y3 & 0.075043 & 0.076785 & 0.086060 \\
x4 & $\mathbf{- 0 . 3 6 0 5 6 0}$ & -0.094028 & -0.007491 \\
y4 & -0.075043 & -0.076785 & -0.086060 \\
x5 & 0.243096 & 0.146147 & -0.039854 \\
y5 & 0.066520 & 0.113644 & 0.062436 \\
x6 & 0.243096 & 0.146147 & -0.039854 \\
y6 & -0.066520 & -0.113644 & -0.062436 \\
x7 & 0.085073 & 0.123375 & -0.059546 \\
y7 & 0.198131 & 0.110449 & 0.150668 \\
x8 & 0.085073 & 0.123375 & -0.059546 \\
y8 & -0.198131 & -0.110449 & -0.150668 \\
x9 & 0.079340 & 0.209158 & $\mathbf{- 0 . 4 4 3 6 1 7}$ \\
y9 & 0.212871 & 0.209860 & 0.163603 \\
x10 & 0.079340 & 0.209158 & $\mathbf{- 0 . 4 4 3 6 1 7}$ \\
y10 & -0.212871 & -0.209860 & -0.163603 \\
x11 & -0.070806 & 0.010241 & 0.367298 \\
y11 & 0.178455 & 0.078143 & 0.242364 \\
x12 & -0.070806 & 0.010241 & $\mathbf{0 . 3 6 7 2 9 8}$ \\
y12 & -0.178455 & -0.078143 & -0.242364 \\
$\mathbf{x 1 3}$ & $\mathbf{0 . 4 5 6 4 1 3}$ & $\mathbf{- 0 . 8 1 1 4 2 0}$ & -0.040131 \\
y13 & 0.000000 & 0.000000 & 0.000000 \\
\hline & & &
\end{tabular}

\section{DISCUSIÓN}

El proceso de formación de nuevas razas o variedades domésticas animales suele realizarse a través de la selección artificial, que aprovecha la variación hereditaria. En la selección de nuevas variedades de conejo toy, el objetivo final es la obtención de "nuevos" animales fenotípicamente únicos y a la vez atractivos (menor tamaño, cara "leonada", pelo largo en el cuerpo, combinación atractiva de colores...), y siempre conservando -o aumentando- el carácter pedomórfico, carácter que hace más atractivo un animal (Borgi \& Cirulli, 2016). Esta pedomorfia representa, al fin y al cabo, un proceso de neotenia "forzada" por el hombre, que provoca que retengan caracteres físicos infantilizados (Skulachev et al., 2017).

El carácter pedomórfico de los toy ha sido recientemente objetivado por métodos morfogeométricos (Parés-Casanova et al., 2018). A la vista de nuestra resultados, aunque no fuese el objetivo de la investigación, se expresa esta pedomorfia como una falta de dimorfismo sexual en cuanto al tamaño, puesto que el conejo doméstico presenta normalmente diferencias craneales diferentes entre sexos (Özkadif \& Eken, 2016). Para el caso de los animales domésticos, la obtención de animales pedomórficos la selección es totalmente artificial. Esta selección, en el caso de los conejos toys, se centra principalmente en los machos, queestarían pues sometidos a una mayor presión de esta selección, en comparación con las hembras.

Tanto los cambios genéticos como ambientales pueden incrementar la asimetría fluctuante, que viene a representar el deterioro en la homeostasis del desarrollo de la morfología adulta debido a una menor estabilidad del desarrollo (Parés-Casanova et al., 2020). Explicaría pues ello que los machos expresen valores de asimetría fluctuante más elevados, asimetría, por otro lado, que estaría localizada básicamente a nivel facial. La asimetría direccional, también detectada en este estudio, podría estar relacionada con una lateralización anatómica y etológica (Uros`evic’ et al., 2015).

El dimorfismo sexual en la asimetría craneal, como expresión de estrés, es de interés porque puede predecir di- 
ferencias en salud, bienestar y comportamiento.Somos conscientes de que nuestro estudio se realizó sobre conejos toy, el tamaño de la muestra es limitado y femenino limitado, por lo que por el momento no es posible extrapolar los resultados a otros animales domésticos ni otro tipo de conejos domésticos (carne, piel...). Pero abre todo ello un nuevo campo de estudio, sobre todo para la valoración del bienestar individual en razas domésticas pedomórficas extremas -no sólo conejos.

\section{AGRADECIMIENTOS}

El autor agradece a CUNIPIC, en Vallfogona de Balaguer (Cataluña) por ceder los cadáveres de sus animales para ser incorporados a la colección osteológica universitaria. También el autor debe expresar su sincera gratitud a los árbitros anónimos.

PARÉS-CASANOVA, P. M. Different skull asymmetry according to sex in toy rabbits. Int. J. Morphol., 39(3):864-868, 2021.

SUMMARY: Toy rabbits are selected for their paedomorphic ('babyish') appearance. The main objective of this study is to analyse the skull asymmetries of toy rabbits. For this purpose, a total of 46 adult skulls ( 9 males and 37 females) were studied. Geometric morphometric technique with five pairs of anatomical landmarks and three on sagittal plane on dorsal aspect of skull was used. Our hypothesis is that through artificial selection on males (which are the main subject for obtaining new phenotypes among toys) asymmetries will be expressed as higher asymmetrical values. Skulls' size was similar between sexes, but males presented a higher level of fluctuating asymmetry. Fluctuating asymmetry indicates a negative ability to buffer subtle developmental anomalies, normally from genetic or environmental origin. In other words, males would be under stronger directed selection and consequently be more affected by management (including selective) factors compared to females. These results can allow a better understanding of the patterns and processes underlying sexual shape dimorphism, especially with extreme phenotypes, as toy rabbits are.

KEY WORDS: Bilateral asymmetry; Oryctolagus cuniculus; Osteology; Paedomorphy; Sexual dimorphism.

\section{REFERENCIAS BIBLIOGRÁFICAS}

Bookstein, F. L. Morphometric Tools for Landmark Data: Geometry and Biology Morphometric Tools for Landmark Data: Geometry and Biology. Cambridge, Cambridge University Press, 1991.

Borgi, M. \& Cirulli, F. Pet face: mechanisms underlying human-animal relationships. Front. Psychol., 7:298, 2016.

Briones, C. \& Guiñez, R. Una revisión de la asimetría bilateral en bivalvos. Rev. biol. mar. oceanogr., 43(1):1-6, 2008.
Goodwin, D.; Bradshaw, J. W. S. \& Wickens, S. M. Paedomorphosis affects agonistic visual signals of domestic dogs. Anim. Behav., 53(2):297304, 1997.

Graham, J. H.; Raz, S.; Hel-Or, H. \& Nevo, E. Fluctuating asymmetry: methods, theory, and applications. Symmetry, 2(2):466-540, 2010.

Hammer, Ø.; Harper, D. A. T. \& Ryan, P. D. PAST: paleontological statistics software package for education and data analysis. Palaeontol. Electron., 4(1):4, 2001.

Klingenberg, C. P. MorphoJ: an integrated software package for geometric morphometrics. Mol. Ecol. Resour., 11(2):353-7, 2011.

Klingenberg, C. P. Studying morphological integration and modularity at multiple levels: concepts and analysis. Philos. Trans. R. Soc. Lond. B Biol. Sci., 369(1649):20130249, 2014.

Lotto, F. P. \& Beguelin, M. Asimetría direccional del postcráneo en poblaciones prehispánicas del sur de Sudamérica. Rev. Mus. Antropol., 7(1):133-42, 2014.

Mardia, K. V.; Bookstein, F. L. \& Moreton, I. J. Statistical assessment of bilateral symmetry of shapes. Biometrika, 87(2):285-300, 2000.

Özkadif, S. \& Eken, E. Craniometric measurements of New Zealand rabbits skull from three- dimensional reconstruction images. J. Anim. Vet. Sci., 2:9-14, 2016.

Palmer, A. R. Fluctuating Asymetry Analysis: A Primer.” In: Markow, T. A. (Ed.). Developmental Instability: Its Origins and Evolutionary Implications. Dordrecht, Kluwer, 1994. pp.335-64.

Parés-Casanova, P. M.; Minoves, J.; Soler, J. \& Martínez-Silvestre, A. Divergent scute asymmetry among pure and crossed individuals of Testudo hermanni (Gmelin, 1789). Arx. Misc. Zool., 18:43-50, 2020.

Parés-Casanova, P. M.; Sofiane, K. \& Medina, A. Diferente desarrollo cefálico según tipo de conejo. Rev. Cienc. Vet., 36(2):15-21, 2018.

Rohlf, F. J. The tps series of software. Hystrix Ital. J. Mamm., 26(1):9-12, 2015.

Skulachev, V. P.; Holtze, S.; Vyssokikh, M. Y.; Bakeeva, L. E.; Skulachev, M. V.; Markov, A. V.; Hildebrandt, T. B. \& Sadovnichii, V. A. Neoteny, prolongation of youth: from naked mole rats to "Naked Apes" (Humans). Physiol. Rev., 97(2):699-720, 2017.

Urosevic, A.; Ljubisavljevic, K. \& Ivanovic, A. Fluctuating asymmetry and individual variation in the skull shape of the common wall lizard (Podarcis muralis Laurenti, 1768) estimated by geometric morphometrics." Herpetol. J., 25(3):177-86, 2015.

Zachos, F. E.; Hartl, G. B. \& Suchentrunk, F. Fluctuating asymmetry and genetic variability in the roe deer (Capreolus capreolus): a test of the developmental stability hypothesis in mammals using neutral molecular markers. Heredity, 98:392-400, 2007

Corresponding author:

Parés-Casanova, P.M.

Department of Animal Science, ETSEA

University of Lleida

Catalonia

SPAIN

E-mail: peremiquelp@ca.udl.cat

Received: 04-01-2021

Accepted: 14-03-2021 